\title{
NCI CTEP SDC Germ Cell Tumors of the CNS Sub-Category Terminology
}

National Cancer Institute

\section{Source}

National Cancer Institute. NCI CTEP SDC Germ Cell T umors of the CNS Sub-Category

Terminology. NCl Thesaurus. Code C103048.

Germ Cell T umors of the CNS is an NCI Cancer Therapeutic Evaluation Program (CTEP) Simplified Disease Classification (SDC) category used to org anize cancer-related disease coding that harmonizes with and supports reporting based on the global standard Medical Dictionary for Drug Regulatory Reporting (MedDRA) terminology. 